\title{
Informal physics programs as communities of practice: How can programs support university students' identities?
}

\author{
Brean Prefontaine $\odot,{ }^{1}$ Claire Mullen $\odot,{ }^{2}$ Jonna Jasmin Güven $\odot,{ }^{3}$ Caleb Rispler, ${ }^{4}$ \\ Callie Rethman $\odot,{ }^{5}$ Shane D. Bergin $\odot,{ }^{6}$ Kathleen Hinko, ${ }^{1,4}$ and Claudia Fracchiolla $\odot^{6}$ \\ ${ }^{1}$ Department of Physics and Astronomy, Michigan State University, East Lansing, 48824 Michigan, USA \\ ${ }^{2}$ School of Mathematics and Statistics, University College Dublin, Dublin, Ireland \\ ${ }^{3}$ Department of Physics and Astronomy, The University of Manchester, Manchester, United Kingdom \\ ${ }^{4}$ Lyman Briggs College, Michigan State University, East Lansing, 48824 Michigan, USA \\ ${ }^{5}$ Department of Physics, Texas A\&M University, College Station, 77843 Texas, USA \\ ${ }^{6}$ School of Education, University College Dublin, Dublin, Ireland
}

(Received 31 August 2020; revised 9 September 2021; accepted 13 September 2021; published 12 November 2021)

\begin{abstract}
Many undergraduate and graduate physics students participate in some form of public engagement throughout the course of their studies, often through groups supported by physics departments and universities. These informal teaching and learning programs can offer unique opportunities for both participants and facilitators to interact in a way that fosters physics identity development. Understanding how physics identities can be cultivated will allow us to work toward a field that is inclusive of more identities. In this study, we build on previous work to investigate facilitator experiences in three informal physics programs using an operationalized communities of practice framework. Through our analysis, we identify different structures within these programs that support physics identity development.
\end{abstract}

DOI: 10.1103/PhysRevPhysEducRes.17.020134

\section{INTRODUCTION}

Informal physics programs, activities, and events are learning experiences that happen outside of the formal classroom. Informal physics programs come in a variety of forms, are made for different audiences, and often have a variety of goals. Additionally, informal learning experiences offer agency to the learner and are free of many of the constraints seen in classrooms, such as standards and grades [1]. Many researchers and practitioners refer to these informal physics environments and events as "outreach" or "public engagement" opportunities [2]. These terms primarily refer to the role that physicists take in these educational activities; in this paper we choose to use the term "informal" to reference the learning environment more holistically and capture all of these different ways of engaging with physics outside of a formal learning environment.

The diversity of activities that take place in informal spaces make them rich opportunities for physics education research. Informal learning environments are important to study because these physics spaces and programs can provide support to students of all ages in many different ways. Often, the research community has focused on the impact that participating in these programs has on the

Published by the American Physical Society under the terms of the Creative Commons Attribution 4.0 International license. Further distribution of this work must maintain attribution to the author(s) and the published article's title, journal citation, and DOI. audience. However, we know that participation in these spaces also has an impact on those who facilitate them, especially undergraduate and graduate physics students [3-7]. In past work, we have found a variety of opportunities for growth and support are available to students which facilitate informal physics programs, such as gaining teaching experience, developing communication skills, increasing content knowledge, and being able to feel like experts within their field of study [7-11].

Additionally, these programs also provide space for student facilitators to explore and develop physics identities [12]. In this paper, we refer to physics identity as the moment in which an individual feels comfortable stating their belonging to the physics community [13-16]. By focusing on how physics identities are supported and fostered within these environments, we can better understand how to support students within their physics studies; additionally, cultivating a physics identity has been linked to persistence within the field $[13,14,17,18]$. An understanding of physics identity is particularly important for students who are historically marginalized and underrepresented in the field of physics because it can lead to a change in seeing themselves as a physicist and their sense of belonging within the field $[15,16,19,20]$. Individual's identities are complex and made up of multiple, intersecting factors (such as race, gender, interest). In part, disengagement or feeling like they do not belong can be connected with tensions in students' intersecting identities [15,16,21-26]. Previous studies have looked at what environments and structures allow students to bring forward their intersecting identities, including their science, 
technology, engineering, and mathematics (STEM) identities, in formal environments [25-28]. In this work, we are interested in the environments and structures within informal spaces that allow students to bring forward their intersecting identities, which include their physics identity. We hypothesize that informal physics programs offer a space for positive physics identity development among facilitators in a way that differs from formal physics education. Informal spaces can allow for individuals' diverse identities to be brought to the practice. Therefore, understanding how structures in informal physics programs impacts university students' physics identity is important for designing supportive learning environments in both informal and formal spaces.

The community of practice framework, originated by Lave and Wenger, looks at identity development from the sociocultural perspective, and therefore serves as a lens to understand the development and support of intersecting identities. The sociocultural premise of community of practice theory, in contrast to a sociocognitive or purely cognitive approach, reflects their extensive ethnographic research into ways that apprenticeship is enacted in various groups. Many empirical studies have been done that apply the community of practice framework as a tool to understand group dynamics and identity formation, especially in the fields of business and education [29-31].

Additionally, previous studies have used the community of practice framework to understand development of physics identity in formal learning spaces. In physics, Close et al. looked at how participation in the learning assistance (LA) program supported university students' physics identity $[32,33]$. They combined the mechanisms of identity from the community of practice framework with Hazari's physics identity constructs to determine what factors from the LA program impacted the students' physics identity [24]. A study by Irving and Sayre used the community dimensions of the community of practice framework to determine if participation in upper division physics labs impacted university physics students' level of membership in physics, and showed shifts in students' perceived identity development [34]. By viewing physics as a community of practice and research as a practice of that community, Irving and Sayre found that students participating in research shifted toward becoming more central members of the community.

In prior work, we have postulated that informal physics programs can support physics identity by functioning as a community of practice for undergraduate and graduate student facilitators $[3,4,6,12]$. We operationalized Lave and Wenger's theoretical communities of practice framework so that it was contextualized to the informal physics environment [12]. We then utilized a case study approach to understand the individual experiences of university students who acted as program facilitators within an informal physics program. In using the communities of practice framework to understand the experiences of the university students, we were able to learn how individuals navigate their membership and the development of their physics identity within these communities.

In this paper, we build on our prior work that operationalized the community of practice framework, and now apply it to multiple informal physics contexts. We do this to investigate if there are different ways that student facilitators may grow in their identities as members of informal physics communities, as people who do physics, and as members of the broader physics community. We also seek to identify structures and norms of informal physics programs that contribute to positive or negative identity development opportunities within these communities of practice. Thus, we seek to answer two questions: In what ways can the identities of student facilitators be affected in informal physics programs that are communities of practice? What are structures and practices of informal physics programs that support physics identity development in physics student facilitators?

To answer these questions, we have conducted an investigation into three different informal physics programs that function as communities of practice [35]. We collected interviews from student facilitators about their motivation and experience participating in the programs. We analyzed these interviews with our operationalized framework for informal physics programs to determine how these programs may support identity development. From this analysis, we also extracted themes spanning the programs about the key structures and norms that support student facilitators. Results from this study are relevant to both practitioners and researchers who are looking to improve the support given to university physics students and when creating informal and formal learning environments.

\section{COMMUNITIES OF PRACTICE FRAMEWORK}

In 1991, Lave and Wenger developed the communities of practice framework to identify and study groups who come together with a shared concern or goal and who spent time together learning how to achieve that mission $[33,36]$. This theoretical framework is a social theory of learning, useful for understanding how members within the group, and the group as a whole, learn and evolve to achieve a shared goal. The group develops a set of norms and practices that members of the group can recognize, practice, and influence in order to become part of the collective. Furthermore, Lave and Wenger used this communities of practice framework to understand how participation impacts identity through membership among participants.

Not every group is considered a community of practice; a group must contain three aspects to be considered a community of practice, as shown in Fig. 1. The group must have some sort of shared goal or proficiency (the domain), interactions between group members that help individuals achieve the goals (the community), and a set of common norms, repertoire, and shared information needed to achieve the goals (the practices) [34]. If a domain, 


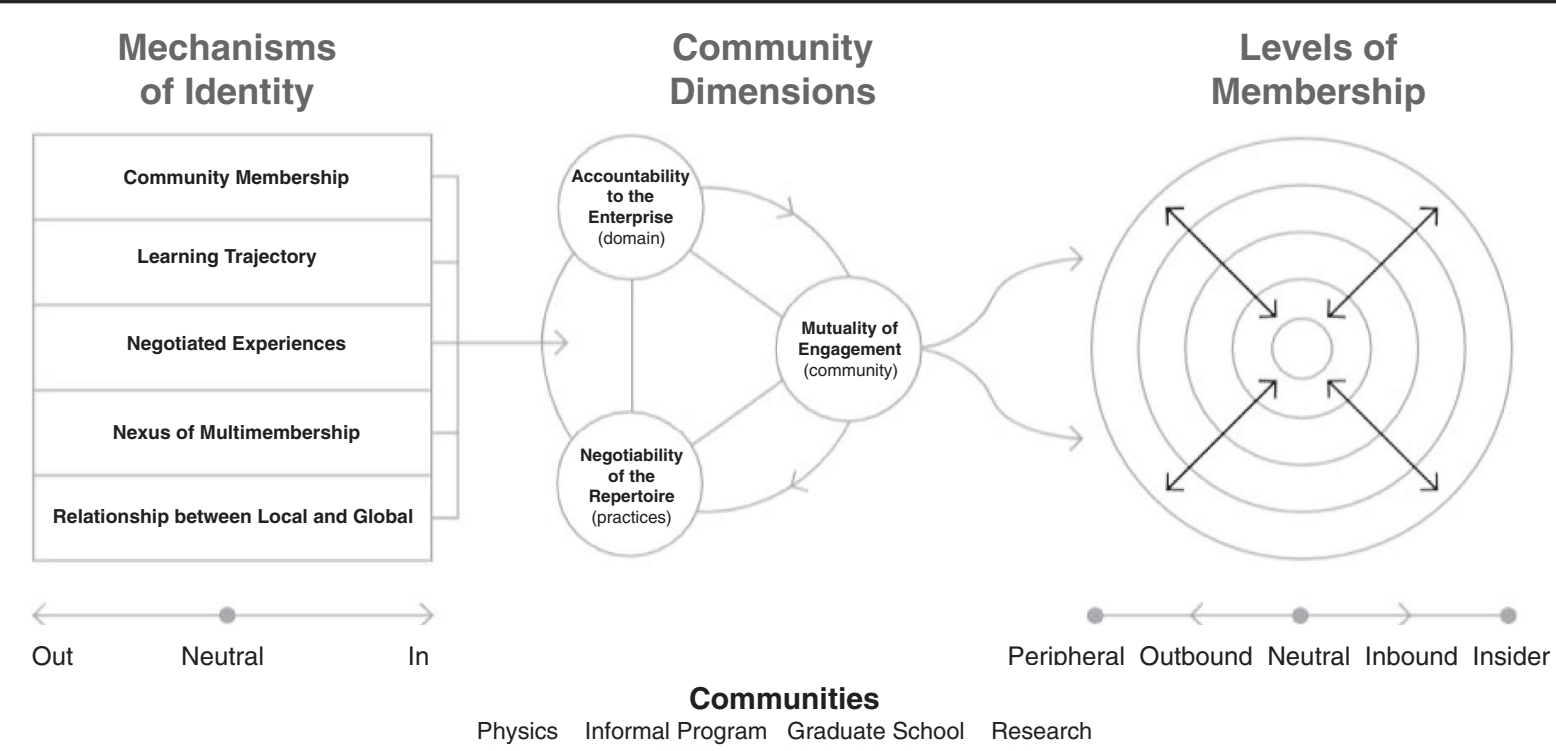

FIG. 1. The three essential elements of a community of practice (shown in the middle column) contain the domain, community, and practice and must all be identifiable within a community in order to use the communities of practice framework. The mechanisms of identity (first column) interact with the elements of the community of practice and influence individuals' membership levels (shown in the third column).

community, and practices can be identified for a group of individuals, then it becomes appropriate to consider the group as a community of practice and to use the framework as an analysis tool.

When thinking about the physics community, we can also identify the three essential elements of a community of practice. Even though there are subgroups based on research topics, projects, and departments, the physics community can be viewed as a group that has a common vision of developing a deeper understanding of the universe's behavior (the domain of the physics community). The community consists of all of the members of the physics community who are interested in working toward achieving that goal. Finally, physics has some common norms and practices, such as the use of mathematics to communicate phenomena or methods used to explore the natural world.

If a group can be considered a community of practice, then it makes sense to consider both the roles individuals take within the community and also how individuals' identities are shaped and shape the community. In prior work, we operationalized these two aspects of Lave and Wenger theory in the context of informal physics programs. Our operationalized communities of practice framework [12] defines (i) community dimensions, which help identify how the three aspects of a community of practice (domain, practice, and community) are recognized and impact an individual's membership in the community, and (ii) mechanisms of identity, which identify the specific structures of informal physics programs that promote changes in an individuals' membership (i.e., if they move from being a peripheral member of the community to being a more central member). There are three community dimensions derived from what constitutes a community of practice (defined in Table I): accountability to the enterprise is the commitment of individuals to the goals (domain) of the community. Mutuality of engagement is the kind of interactions that an individual has with other members (community), and negotiation of the repertoire is how an individual understands and engages in the activities of the group (practices) [34,37]. These dimensions allow us to determine the role individuals perceive they have within the community of practice.

Additionally, Wegner's framework identifies a bidirectional connection between a community of practice membership and identity development: "Building an identity consists of negotiating the meanings of our experience of membership in social communities" [36]. Because of the established connection between the membership of a community of practice and identity, Wegner determines that experiences within the community of practice that sustain a membership are then in turn fostering identity development. Therefore, through the mechanisms of identity constructs (defined in Table II) we understand what exactly is contributing to changes in the individuals' membership. Negotiated experiences are the ways individuals make meaning of experiences in the community. Community membership is an individual's development of forms of competence and performance valued by the community, such as norms of interactions and the use of shared practices and resources. Learning trajectory marks events that have taken place in the past or things that have been learned that resulted in different forms of participation within the community. Nexus of multimembership highlights how members coexist and participate in different 
TABLE I. The three community dimension constructs with definitions and examples from the communities of practice framework.

\begin{tabular}{|c|c|}
\hline Community dimensions & Definition \\
\hline $\begin{array}{l}\text { Accountability to the } \\
\text { enterprise }\end{array}$ & $\begin{array}{l}\text { An individual's commitment and understanding of the goals and mission of the community. For example, } \\
\text { talking about wanting to share physics with younger students. }\end{array}$ \\
\hline Mutuality of engagement & $\begin{array}{l}\text { Interactions an individual has with other members of the community, such as peers, audience members, or } \\
\text { directors or coordinators; also highlights how the individual is recognized or influenced by other community } \\
\text { members. For example, talking about having fun driving to events with their friends in the group. }\end{array}$ \\
\hline $\begin{array}{l}\text { Negotiability of the } \\
\text { repertoire }\end{array}$ & $\begin{array}{l}\text { An individual's understanding of the practices of the community, including specific activities that the } \\
\text { community performs to reach their goals and knowledge that the members need to have in order to be } \\
\text { successful. For example, learning new demos using pressure to expand marshmallows. }\end{array}$ \\
\hline
\end{tabular}

communities. Relationship between local and global is an individual's negotiation of the sense of belonging within the local community and how that fits into a broader sense of the more universal community [34,37].

By considering both the community dimensions and mechanisms of identity, our operationalized communities of practice framework allows us to understand how membership of university students evolves throughout their facilitation of informal physics programs. Through participation over time, students may become more central members of the community; alternatively, if their level of commitment lessens, they may become a more peripheral member. To identify membership levels, we turned to how Lave and Wenger discuss membership. The original communities of practice framework identified five membership levels-central, insider, neutral, peripheral, and outsider [34]. Parting from this element of their theory in order to make the coding more consistent, we created a set of subcodes during the operationalization process that identifies membership levels for each community dimension and the type of changes mechanisms of identity promoted. in our framework, the community dimension constructs have five subcodes identifying membership levels-insider, inbound, neutral, outbound, and peripheral. Similarly, the mechanisms of identity have three subcodes that identify movement between membership levels-inbound, neutral, and outbound. Members of a community of practice who are thoroughly involved in the goals and practices of the community would be considered a member of the insider level. Likewise, a member who is only tangentially involved with the community or who is just starting to learn about the community would be considered a member of the peripheral level. An individual's membership level is dynamic and so a member can move toward more central positions within the community or more external positions. For example, someone who has enjoyed participating and expresses the desire to continue participating in a certain community would experience

TABLE II. The five mechanisms of identity constructs with definitions and examples from the communities of practice framework.

Mechanisms of identity

Negotiated experience

Learning trajectory

Nexus of multimembership

Community membership

Relationship between local and global
Definition

The process of an individual making meaning of experiences through participation in the community and interactions with other members of the community. These experiences are what allows them to develop an identity as a member of the community. For example, talking about how interacting with audience members during a physics event is really exciting.

Events that have taken place in the past or things that have been learned that resulted in different forms of participation or changes in membership within the community. For example, talking about physics camps that they did as a young kid and wanting to do something like that again.

The participation and interactions of individuals with multiple communities, including the targeted community of practice, which requires coordination and negotiation. This construct highlights all forms of participation that contribute to our complete mesh of identities and how members coexist in different forms of membership. For example, talking about how it is hard to have enough time to do physics outreach and physics research.

The development of forms of competence and performance valued by the community, such as norms of interactions and the use of shared practices and resources. This mechanism identifies how more central members of the community become perceived as competent by other members and personally feel competent. For example, talking about wanting to run in an election for a leadership role within the group.

The negotiation of the sense of belonging within the local communities and how that fits into a broader sense of more universal community. For example, talking about how physics outreach allows them to show younger girls how science can be exciting. 
identity development toward more central membership within the community (inbound), while someone who is not as deeply involved in the core activities may experience a shift toward a less identity development (outbound). Members who continue to be involved in the community at the same level may not experience a shift in identity development (neutral). A more detailed discussion of the communities of practice framework, the operationalized framework, and our methods of validation can be found in our previous work [12].

\section{ESTABLISHING INFORMAL PHYSICS PROGRAMS AS COMMUNITIES OF PRACTICE}

In this study, we wanted to apply the operationalized communities of practice framework to several different types of informal physics programs in order to document different ways that informal physics groups may provide opportunities for identity development [12]. In selecting our cases, it was a necessary step to determine if an informal physics program did in fact have the characteristics of a community of practice before applying the framework to analyze group members' experiences. Informal physics activities can range widely in terms of format, frequency, content, audience, and level of involvement of university physics students; thus, it is probable that not all informal physics efforts can be classified as a community of practice. For example, a common type of physics public engagement event is popularized lectures aimed at high school students or adults. Often these lectures are given by individual faculty with little extended involvement from university physics students or others within the department-it is less likely that these efforts could be considered communities of practice such as the ones that we are considering. In contrast, we are particularly interested in informal physics efforts that involve many members all working toward the same goal.

The programs selected for this study are Science Theatre (based at Michigan State University), PISEC (based at the JILA Physics Frontier Center at the University of Colorado Boulder), and Quavers to Quadratics (a collaboration between University College Dublin, Trinity College Dublin, and the Irish National Concert Hall). ${ }^{1}$ All three programs were chosen because they have large numbers of physics student volunteers, have been active for a number of years (Science Theatre since 1991, PISEC since 2008, and Quavers to Quadratics since 2014), and have close links with university physics departments. Furthermore, the design, content, and implementation of the three programs provide a range of formats, from the more common (demonstration show) to the more novel (blending physics and music). They also range in their involvement of physics

\footnotetext{
${ }^{1}$ You can find more information about Science Theatre at web .pa.msu.edu/sci_theatre, Quavers to Quadratics at www.nch.ie, and about PISEC at www.colorado.edu/outreach/pisec.
}

students, as well as the different types of students, with one group being entirely organized by undergraduate students (Science Theatre), one group being mostly physics graduate students (PISEC), and one group bringing physics students together with music and education students (Quavers to Quadratics). Thus, these three programs allow us to gain insight into a wide, international variety of informal physics programs that utilize university students as facilitators within the program.

Additionally, an important consideration in the selection of programs was that at least one of the authors has been, or is currently, a practitioner associated with a program in some capacity. Our direct knowledge of these specific programs served to validate the selection of these programs as candidates for communities as practice; it further acted as a validation measure for data analysis [38,39]. Another affordance of our practitioner experiences is that they provided us with insight in our interpretation of how the community of practice analysis connected with the structures and norms of programs. This interpretation is described in Sec. VII and is an important bridge connecting research to practice in this work.

Below are short descriptions of the format and content of each program in this study. A domain, community, and practices were identified for each program (more specific details are given in the Supplemental Material [40]). Broadly, the domain of each program was centered around university students achieving a common goal of sharing physics knowledge with youth outside of the field. The specific practices of each program differ considerably since the format of each program is different. However, all three programs involve university physics students taking some form of leading interactive physics activities with youth.

The community of each program consists of all the student facilitators, staff, and faculty who work together to develop and deliver the program content. One significant consideration here is whether to include the youth participants as part of the community as well. In Science Theatre and Quavers to Quadratics, youth typically interact for only a short period of time with the undergraduate facilitators and mostly receive the physics content by interacting with already developed demonstrations - therefore, we did not include them in the community. However, in PISEC, youth and facilitators meet in small groups weekly over the course of a semester or often an entire year, and they are encouraged to co-create new activities. Because of the extended relationships that are formed in this program, we include youth as part of the PISEC community.

Quavers to Quadratics is a program for school children to play with ideas common to physics and music. The children (typically 8 to 12 years of age) are led in their play by undergraduate students from physics, music education, and science education. Quavers to Quadratics is a collaboration between a national cultural institution (the National Concert Hall) and two universities in Ireland (author Shane 
Bergin is one of the founders and directors). University students from three departments (physics, science education, and music education) at the corresponding universities participate in the program. One of the aims of Quavers to Quadratics is to have a creative space for undergraduate students to play, work with others from outside their disciplinary niche, and to gain teaching experiences such that they might reflect on their view of physics, music, and education (domain). The program runs twice a year with 15-20 undergraduates a semester, who are paid for their effort (community). Each three-month cycle requires the undergraduate students to co-plan, co-teach, and co-reflect upon two initial classroom visits at the children's school, a day-long workshop at the National Concert Hall, and a final classroom visit. In these interactions, facilitators encourage children to explore questions like "what is a wave?" "how does frequency and amplitude of a wave affect sound?", and "how do the shapes and sizes of musical instruments affect sound?" (practices).

Science Theatre is an undergraduate student group that performs physics and other science demonstrations with a theatrical twist. Along with reaching younger audiences (typically 5-17 years old), Science Theatre aims to provide participants with a space to pursue their passions for physics or science and public engagement with like-minded others (domain). It is supported by the Department of Physics and Astronomy financially and has a faculty advisor (author K. H. has helped in this role). The majority of students are undergraduates majoring in physics or other STEM fields (community). Throughout the year, the student organization partners with science events and local schools to perform stage shows and bring hands-on activities for elementary and middle school kids. They do physics demonstrations and activities (such as bed of nails and a flame tube) as well as some other science demonstrations (practices). The group devotes each spring break to a road trip that aims to visit as many schools as possible in the rural upper peninsula of Michigan. The group has an officer system with twelve elected roles and holds frequent general body meetings.

PISEC (partnerships for informal science education in the community) is an after-school program, where physics students work with children to explore fun, hands-on physics activities. This program is the main public engagement effort of the JILA Physics Frontier Center for Atomic, Molecular and Optical Physics at the University of Colorado Boulder. (Authors C.F. and K. H. have served as program directors.) The main goal of PISEC is to give children the opportunity to explore pathways to physics and other STEM careers by engaging them in interactive, inquiry-based physics activities. PISEC also seeks to improve the pedagogical and communication skills of university physics students (domain). It runs each semester with 15-20 volunteers who are mostly physics graduate students. Volunteer facilitators meet with elementary and middle school children once a week for an hour at the children's school (community). During that after-school hour, the children are encouraged to engage in scientific practices through proposing hypotheses, designing their own experiments, and reporting their findings and ideas (practices).

By identifying these three essential elements of a community of practice for each informal physics program using a practitioner-researcher approach, we have established that all three cases have the necessary characteristics of a community of practice. While the three programs being considered in this work are unique in that they involve undergraduate facilitators interacting with children through physics activities, there may be many other informal physics programs that also contain the elements of a community of practice. This finding is of itself important, as it demonstrates that a diverse range of informal physics programs can serve as an environment for physics students to build identity.

\section{UNDERSTANDING STUDENT EXPERIENCES IN INFORMAL PHYSICS COMMUNITIES OF PRACTICE}

In this paper, we use a case study to understand how the domain, community, and practices of each group affect student experience, and how in turn affect student identity development. A case study approach allows us to gain an in-depth and contextualized understanding of facilitators' experiences in the informal physics programs, as well as explore the characteristics, structures, and implications of facilitating informal physics programs for students' physics identity [41]. We followed an interpretative approach to the case study to understand from the individual's point of view the social meaning and participation. To this end, we conducted interviews with students leading these programs (facilitators). Interviews were an appropriate data source because we were looking to explore facilitation experiences through the individual participants' lens alongside their own positionality within their different communities. Interviews were analyzed using the community dimensions (Table I) and mechanisms of identity (Table II) from the operationalized community of practice framework.

\section{A. Interviews with university facilitators}

A semistructured interview protocol was designed to ask participants to discuss their experiences in the program and their perceptions toward physics and informal physics programs. Examples of the questions include: Why did you decide to volunteer for the program? Why would you (or would you not) volunteer again? Do you identify as a physicist? Why did you decide to pursue studies in physics? These questions were meant to prompt the interviewee to talk about how they felt positioned within the program's community of practice and the physics 
community of practice, as well as what experience had led them to initially and continue engaging in those communities. The individual's answer would connect the experiences to their membership within the communities of practices discussed and therefore their identity. The semistructured interviews allowed us to have the freedom to follow up on questions and be able to capture as much of the narrative as possible. Interviews were conducted by three researchers and lasted about an hour on average. Additional details about the interview protocols can be found in the Supplemental Material [40].

There were three variations of the protocol, each adapted to the corresponding program. However, all three were centered on questions about the student facilitator's motivations for participating in the programs, the expectations and hesitations regarding volunteering, as well as how the students identified themselves regarding their degree. The differences in the protocols consisted mainly of questions pertaining to the particular practices and/or activities of the relevant program. Other differences in the protocols arose from the time when the interview took place. For example, some students were interviewed once-after they had participated in the program for at least one semester, while other students were interviewed twice during their participation in a program. This difference in frequency and timing of the interviews was mainly due to the nature of the programs. For example, two of the programs require a semester long commitment (PISEC and Quavers to Quadratics) whereas the third program (Science Theatre) allows for students to participate when time allows. Also, two of the programs, Quavers to Quadratics and Science Theatre, offer a week-long trip during spring break or summer break. In the event that one student had completed two or more interviews, we viewed the collection of interviews as a snapshot of the student's relationship with the program. For more details on the interview protocol see the Supplemental Material [40].

We collected interviews from 58 participants across the three informal physics programs. These participants were from the pool of those facilitating the activities in the programs and that were willing to participate in the research study. Based on a careful reading of all interviews by multiple members of the research team, we focused on a subset of 18 participants, which correspond to 29 total interviews. Another 4 participants' interviews were used in our previous paper that explored the use of the community of practice framework [12]. The reason for this selection was threefold: (i) identify the interviews that had descriptive information - some of the interviewees did not provide detailed answers which limited what we could learn from their experiences; (ii) allowed similar representation from the three programs because we had many more interviews from one of the programs than the other two; and (iii) we selected interviews that represented the spectrum of facilitators in the programs - female or male, undergraduates and $\mathrm{Ph} . \mathrm{D}$. students, and postdoctoral fellows - and that the representation reflected the demographics of each program. Seven participants were from PISEC (7 interviews), five participants were from Science Theatre (10 interviews), and six participants were from Quavers to Quadratics (12 interviews). There are several justifications for using a subset of the full data set for analysis: (1) Consistency of interview protocols - earlier versions of the PISEC protocol differed from the protocols used for the other two programs, because we used PISEC participants to test the different protocols. We conducted three different test rounds of the protocol before arriving at the final version, which is the one used in this study and (2) interviews used in earlier stages to operationalize and validate the framework [12] were excluded from the current study. Each interview within our entire dataset was read, or listened to, by members of the research team and the final interviews selected for this paper were intentionally chosen to represent the diversity of the facilitator population within the programs. For more details on the study participants see the Supplemental Material [40].

The interviews used for this paper were conducted by three female researchers: one white woman and two women of color. Interviews were conducted locally, that is, the researcher conducting the interview was, at the time of the interview, based in the institution where the program is hosted. The large majority of the interviews were conducted in person using audio recordings. The length of the interviews varied between $30 \mathrm{~min}$ to an hour, depending on how much detail the interviewee gave in the questions and some of the variations in the protocol.

\section{B. Coding the interviews}

In order to understand what structural elements of each informal physics program contributed to fostering a physics identity among the university student facilitators, we used our operationalized communities of practice framework to analyze the interviews [12]. Narrative inquiry was used to understand participant experiences in the informal physics programs by looking at the content presented in the interviews as well as the language used in the interviews. During the coding process, we used the community dimensions (first layer of coding) to establish membership and the mechanisms of identity (second layer of coding) to determine the agents of change for the membership levels. To understand levels of membership within the community, we used our first layer of coding and assigned one of the five subcodes (insider, inbound, neutral, outbound, and peripheral) to any portion of the interview coded with a community dimension code. The subcodes denote position within membership levels. This position can be static, in the cases of insider or peripheral, or dynamic in the cases of inbound or outbound. A neutral code indicated that from the coded section we could not determine present membership 
levels. For example, if a portion of the interview was coded as accountability to the enterprise and the interviewee was describing how their understanding of the community's mission was evolving, then that same section of the interview would also be coded with the inbound subcode.

Additionally, the second layer of coding was used to identify the mechanisms of identity. The subcodes for the mechanisms of identity indicate whether the particular mechanism being coded prompted a movement inward or outward in the levels of membership. In some instances, the same interview segment could be coded with community dimension and mechanism of identity, however a coded segment cannot be assigned two or more codes from the same layer (i.e., the same portion of the interview could not be assigned accountability to the enterprise and mutuality of engagement). In later sections we denote which code was assigned to each quote that is shared in this paper.

The third and final layer of coding included assigning the community codes. These codes showed which community or communities of practice were being discussed by the interviewee at that instance of the interview. The list of communities discussed throughout this data set included the three informal programs, the student's discipline (usually the physics community), and the graduate school community. Any quotes shared in this paper were coded with one of the program communities and often other communities as the facilitators' experiences in one community could impact their participation in another community.

An example of this coding process can be seen in a quote from Lily, a physics graduate student:

I mean I love physics and I love science, but it sort of makes me even more excited about it, being there, like working on it with the kids and kind of like talking about physics concepts in really simple levels kind of makes it more exciting to me, like "wow this stuff is awesome."

So in that sense I would say they had an impact on me.

This quote was coded as inbound accountability to the enterprise and inbound negotiated experiences because by engaging children in physics and demonstrating to them that physics is fun (part of PISEC domain), Lily is at the same time reiterating her passion for physics.

\section{Coding validation process}

The interview coding process required many members of the research team and was carried out in two main phases: a training and validation phase (where only a few interviews were coded) and an independent coding phase (where the remaining interviews were coded). This two-phase approach allowed us to ensure that each member of the research team understood the framework and felt comfortable coding the interviews. In addition, this phased approach allowed us to utilize inter-rater reliability to ensure that the coding was consistent among all of the interviews and that the codebook could be used by other researchers.

The first phase of the coding process was focused on validation of the codebook and training each member of the research team. First, we had two members of the research team independently code an interview for one program with a set of codes (community dimensions or mechanisms of identity) without the subcodes or communities. The team focused on coding complete ideas and were not too concerned about chunk size. For example, a code could vary in length from a few phrases to two sentences if the same idea was being discussed. Then, the researchers compared their coding in order to discuss and reconcile any differences. This part of the process was meant to check how every part of the interview was coded, not only which codes were used but also the length of the coded segments. If there was a discrepancy about the code used, then there would be a discussion in which each researcher would explain why the corresponding segment was assigned the particular code. Throughout this process, we updated and created a robust codebook with our operationalized framework. Once each of the researchers had a good understanding of the coding framework, a second interview was coded independently. The researchers then compared the codes and estimated kappa value for the interrater agreement value. Once agreement of over $80 \%$ accuracy had been reached, then the researchers independently coded the interviews with the corresponding subcodes and communities. The same process for coder agreement was conducted for each program and for each set of researchers. Cohen's kappa values are given for all subcodes in the Supplemental Material [40].

The second phase of coding was planned to ensure that all interviews were coded and that there was discussion between the researchers during this process. First, we split into teams so that all six of the researchers coding would only focus on one set of constructs (i.e., each researcher only coded for the community dimensions or the mechanisms of identity during this phase). Each researcher was in charge of a set of interviews for coding with the corresponding set of codes and independently coded those interviews. Their partner would then independently code one of the interviews in the set with the corresponding codes to check for interrater reliability. If differences arose, those were discussed with an external researcher member overviewing the process, and the differences were reconciled. Once agreement was more than $80 \%$ accurate then the rest of the coded interviews are reviewed by the partner without having to code independently the interview. This team approach allowed us to ensure that all of the interviews were coded in a timely manner and that interrater reliability was checked for each researcher. 


\section{Statistical analysis methods}

To interpret the data from individual interviews and allow for comparison between the programs, the counts (frequency) of all of the codes from each interview were normalized, i.e., we used percentages to report findings. For each interview, the percentage of each individual code is given with respect to the total number of codes in the interview. For example, in order to calculate the percentage of accountability to the enterprise for an interviewee from Quavers to Quadratics, we divided the frequency of accountability to the enterprise codes for the total frequency of community dimensions codes within that interview. It is important to note here that for the purpose of this study we only looked at the codes that were associated with the program's community of practice. For example, for interviewees from Quavers to Quadratic we only looked at the community dimension and mechanisms of identity codes that were coded under the Quavers to Quadratics community. More details about the reported statistics, including what statistical tests were conducted, can be found in the Supplemental Material [40]. We used statistical analysis to establish a baseline for comparison across the programs. However, as mentioned before, the richness of the data is not only shown through statistical differences but within the narratives. Within the following interview analysis section, we discuss how in some cases the frequency of the code did not reflect the significance of the experience in fostering identity but the significance comes through the stories the facilitators share. This is particularly evident in the analysis section of intersection between community dimensions and mechanisms of identity.

\section{INTERVIEW ANALYSIS}

We want to understand how structural similarities and differences between the programs impacted the community of practice and thus the identities of student facilitators. In order to do this, we look at the community dimensions and mechanisms of identity codes for each program. We also look at the overlap between these layers of codes; this intersection provides information on whether facilitators' membership within their respective community of practice changed throughout their participation in the community and what aspects of the corresponding program's design may have prompted such changes.

\section{A. Community dimensions}

From the overall community dimension codes, we can understand how students perceived their positionality within the programs as well as which dimensions impact their positionality. Figure 2 shows the community dimensions aggregated for each of the three informal programs. One finding to note is there are no statistically significant differences between any of the dimensions for Quavers to Quadratics and Science Theatre. In the case of these two programs, negotiability of the repertoire-which is related

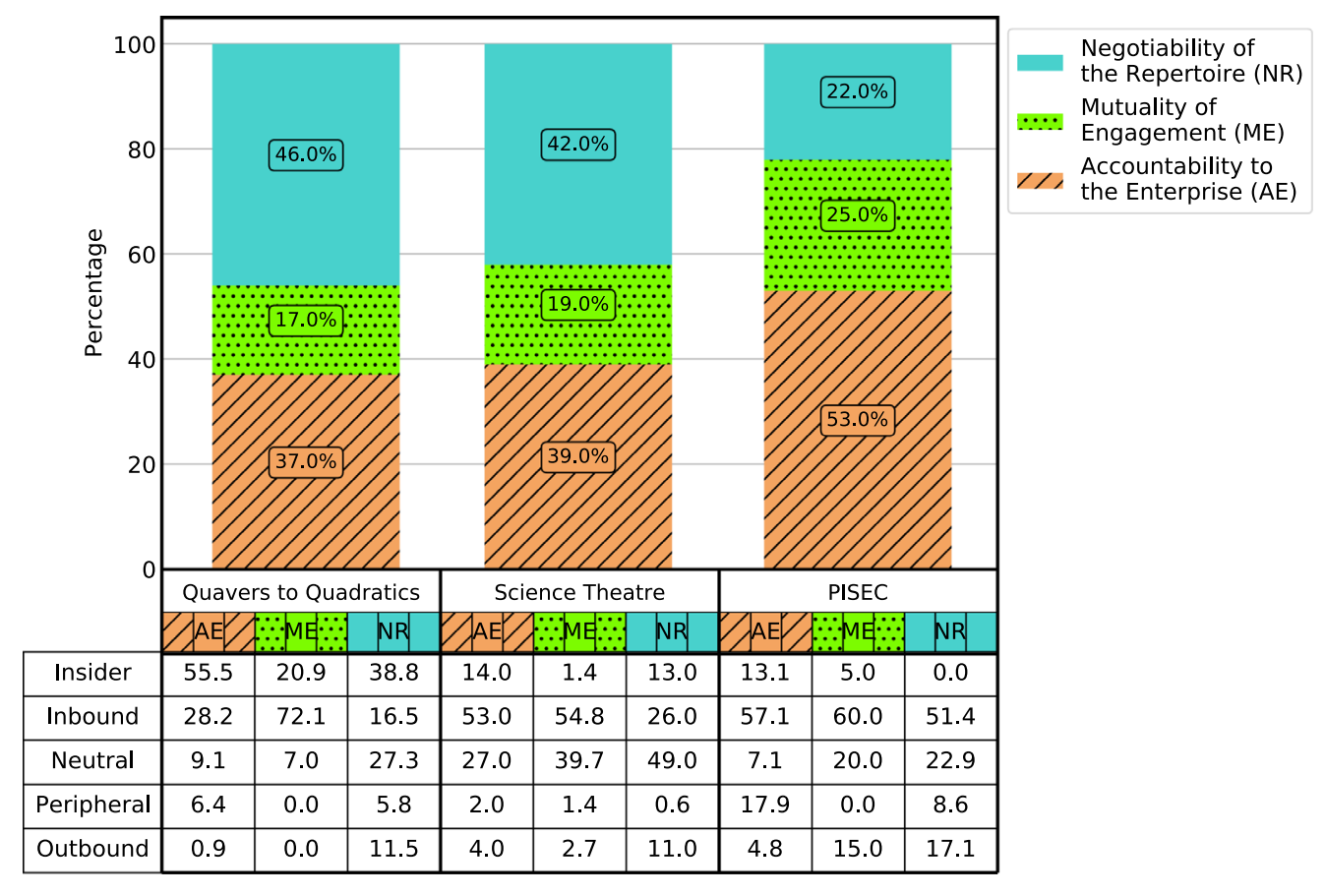

FIG. 2. The overall community dimension codes for each of the three informal programs (left to right: Quavers to Quadratics, Science Theatre, PISEC). The percentages shown in each bar indicate how many of the codes were assigned to each community dimension out of the total community dimensions codes per interview, and thus all the bars add up to $100 \%$. The table shows the breakdown of each community dimension into the subcodes with the percentage indicating how often the subcode was assigned to a given community dimension. 
to knowledge of and competence in the practices of the community - represents more than $40 \%$ of the community dimension codes for both programs, making it the most important factor to establish membership. This emphasis indicates that facilitators in Science Theatre and Quavers to Quadratics measure their membership mainly through their knowledge and competence of the repertoire. In contrast, this mechanism is the least represented community dimension for PISEC (22\%).

The second most coded community dimension for facilitators in Quavers to Quadratics and Science Theatre is accountability to the enterprise, which represents more than $35 \%$ of the codes. We interpret this to mean that to become a central member of the community, students feel they need to actively participate in the practices of the community (negotiability of the repertoire) and have a clear understanding of the mission of the community (accountability to the enterprise).

In contrast, mutuality of engagement represented less than $20 \%$ of the codes for these programs, indicating that connecting to other program facilitators is less important for students in these groups. The main difference in the community dimension codes between these two programs is seen in the subcode percent (see table in Fig. 2). Science Theatre facilitators had higher percentages of neutral codes and Quavers to Quadratics had more insider and inbound codes across all categories. More insider and inbound codes for Quavers to Quadratics indicate that the facilitators perceive that they are moving to become a central member of that community.

In contrast to the other two informal programs, for PISEC, accountability to the enterprise makes up 53\% overall of the community dimension codes. Thus, PISEC facilitators perceived the commitment to the goals (domain) of the community as the leading factor for building membership. The majority of the facilitators had a clear alignment with the values of the program and were demonstrating a strong commitment to the program, shown by a majority of inbound codes within this dimension. Mutuality of engagement makes up 25\% of the codes for PISEC, indicating that connecting to other program facilitators and children is a more important factor for students in PISEC than in the other two programs. Negotiability of the repertoire is the least frequent code (22\%), which shows that PISEC facilitators view understanding the practices as the least important aspect of being a member of the group. Reasons for these differences are due to the different structures of the PISEC program and who constitutes the community. These will be expanded on in the next section.

\section{B. Mechanisms of identity}

While the community dimensions indicate facilitators' perceived level of membership within the community of practice, the mechanisms of identity point out how the particular structures and practices of the program might foster movement in perceived membership levels. Figure 3

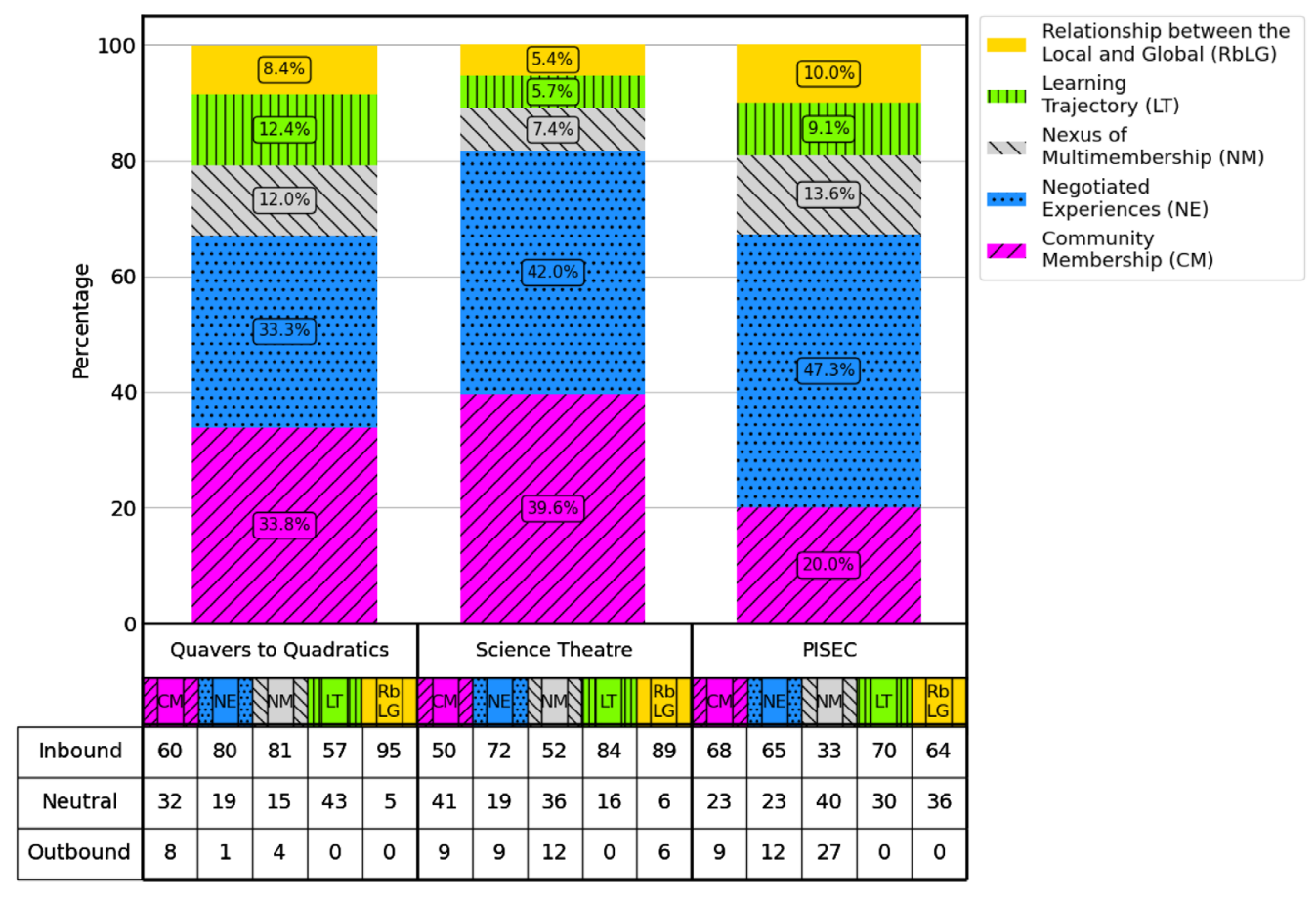

FIG. 3. The overall mechanisms of identity codes for each of the three informal programs (left to right: Quavers to Quadratics, Science Theatre, PISEC). The percentages shown in each bar indicate how many of the codes were assigned to each mechanism of identity out of all mechanisms of identity, and thus all the bars add up to $100 \%$. The table shows the breakdown of each mechanism of identity into the subcodes with the percentage indicating how often the subcode was assigned to a given mechanism of identity. 
shows the mechanisms of identity code distribution for each informal program. Across all three programs negotiated experiences and community membership are the most salient mechanisms, suggesting interactions and connections made through participation while engaging in the practices of the community support membership and identity development. Differences arise among the programs when we look at the mechanisms of identity (as compared to the community dimensions). While all three of the programs have similar missions (domains), each has a unique way of carrying out this mission. This variety of program structures is reflected among the mechanisms of identity distributions, which will be discussed in detail in the following sections.

\section{INTERSECTIONS BETWEEN COMMUNITY DIMENSIONS AND MECHANISMS OF IDENTITY}

In order to gain a more thorough understanding of facilitator experiences within each program, we now look at the interactions of the mechanisms of identity with the community dimension codes. These intersections give insight as to which structural elements within the programs impact facilitator perception of their membership and their identity.

To map the intersections, we looked at the overlap in counts between the mechanisms of identity and each community dimension. We then graphed these values on a pseudoscale that divides the interaction between mechanisms of identity subcodes and one of the community dimensions into four quadrants (see Figs. 4-6). The $x$ axis has subcodes for the community dimensions going from left to right, ranging from outsider to insider. The $y$-axis scale was determined by the mechanisms of identity, ranging from outbound to inbound, going from bottom to top. (See the Supplemental Material [40] for more details). Different shaped markers were used for each of the mechanisms, with the size of the markers representing the normalized frequency (percentage) of the interaction. Plotting the data in this way allows us to visualize which mechanisms are most prevalent with respect to the inbound or insider community of practice experiences (and likewise outbound or outsider experiences). For example, the markers in the upper right quadrant (region shaded green on the table) indicate mechanisms of identity-inbound or community dimensionsinsider interactions and markers in the lower left quadrant (region shaded red on the table) show mechanisms of identity-outbound or community dimension-peripheral interactions. Below, we discuss the key interactions (seen by the larger markers in Figs. 4-6) for each of the three community dimensions through quotes from the facilitators (using pseudonyms) to narrate which aspects of program design impacted the facilitator's identity.

\section{A. Intersection with accountability to the enterprise}

Figure 4 shows how accountability to the enterprise intersects with all five of the mechanisms of identity

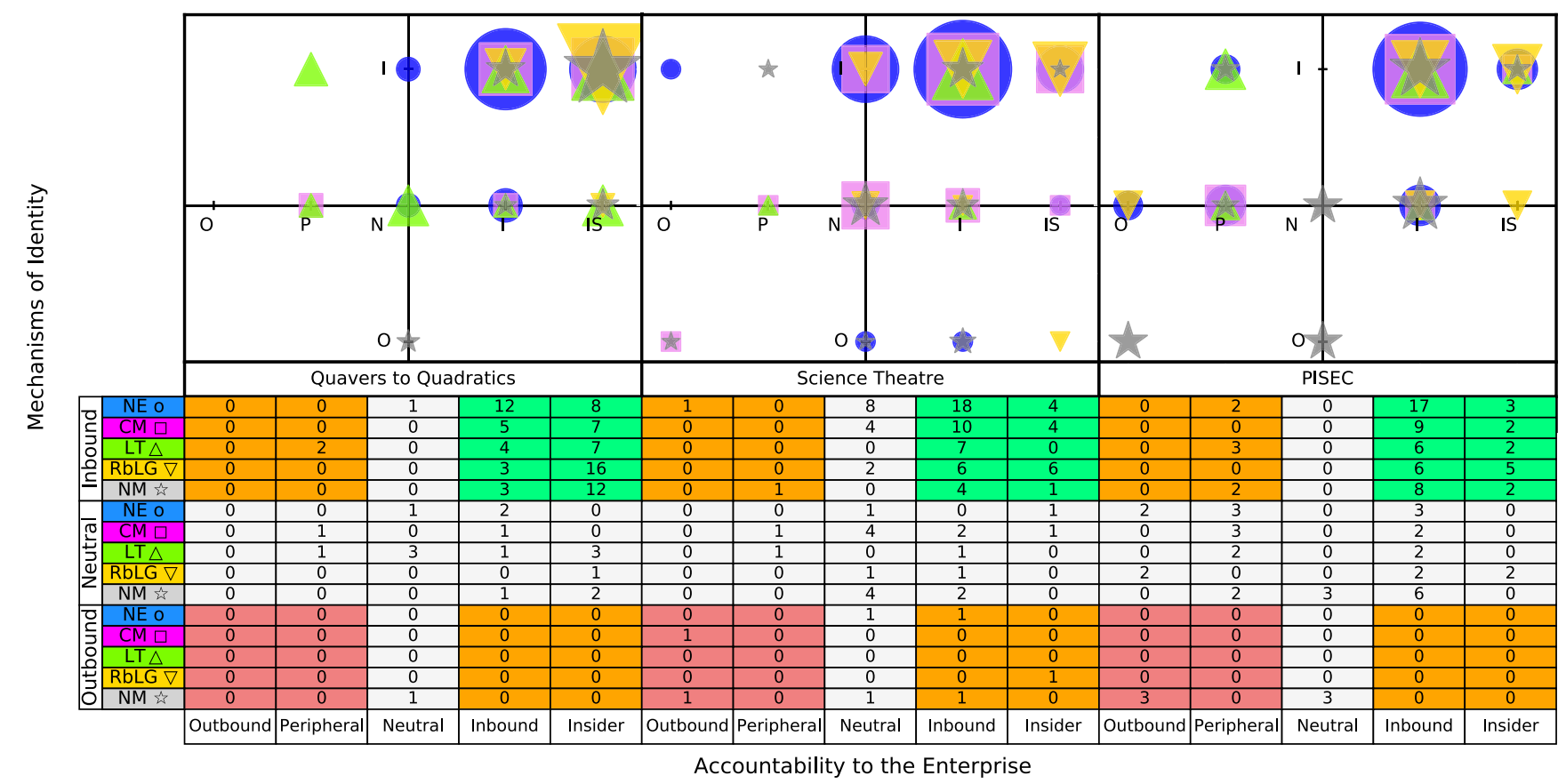

FIG. 4. The intersection of the accountability to the enterprise-community dimension subcodes ( $x$ axis) with all five of the mechanisms of identity and their subcodes ( $y$ axis). Each mechanism is represented by a different color and shape as shown in the second column of the table. The frequencies of the overlaps are shown by the size of the shape and the exact percentage in the table below which follows the same layout as the graph with the four quadrants colored (green: inbound or insider with inbound, orange inbound or insider with outbound or outbound or peripheral with inbound, red: outbound or peripheral with outbound). 
constructs in each of the three programs. Having all five mechanisms of identity interact with the accountability to the enterprise demonstrates that facilitators see an alignment of their values and commitment to the mission of the program and how that impacts all areas of their membership. We consistently saw that the connection with the domain (mission) of the community was a significant aspect of a facilitator's choice to participate in an informal physics education program. This can be appreciated in the upper right-hand quadrant for all three programs in Fig. 4.

The high level of commitment from the facilitators is seen through the high percentage of the inbound and insider subcodes within the accountability to the enterprise code (more than $67 \%$ for all programs) shown in the upper-right quadrant of Fig. 4. However, we also see that community membership, learning trajectory, and relationship between local and global are the mechanisms that interact with this community dimension most frequently. We discuss some of these interactions in the context of the programs below (with more information in the Supplemental Material [41]).

Negotiated experiences (shown by the blue circle in Fig. 4) is seen frequently interacting with accountability to the enterprise and accounts for the majority of the inward movement in membership among facilitators. For example, in PISEC, the connection with children is more impactful than those with other peers, community partners, or members of the community as they build long-term relationships with the children and seeing the effect on their lives made the facilitators "hopeful" and really enjoy the experience. This was explicitly stated earlier as Lily, a physics graduate student volunteering in PISEC, discussed how interacting with the children increases her excitement about physics (as seen in the quote included in Sec. IV B).

Similarly, for Science Theatre the common themes from the interactions of accountability to the enterprise and negotiated experiences are related to how the facilitators enjoyed the children being really engaged, asking questions, getting involved, and expressing that they think science is cool. Tom, an undergraduate student, shared when asked about his experience with children reacting to the demos:

And then, being able to share the science to little kids is really awesome, 'cause I love science, so having the idea that I might inspire a little, or a kid, to even just think something that I did was cool, that's science related.

This quote was coded as inbound accountability to the enterprise and inbound negotiated experiences because, like Lily from PISEC, Tom enjoys the children's positive reaction to the shows and the idea that he might be inspiring children to continue participating in physics or science (Science Theatre domain). These ideas make participation in the Science Theatre community valuable to him and therefore supports his inward movement in the community. Additionally, it reaffirms his passion for science-supporting his science identity.

The engagement and excitement children show while engaging in the activities is a source of motivation and inspiration for many of the Quavers to Quadratics facilitators. Emer, a science education major, was asked if she thought she was having an impact on the children when she shared:

I definitely feel like I'm making some sort of meaningful connection which, like, is such a great thing to see because that's obviously the aim of it... And when I see the kids thinking: 'Oh my God this is amazing! I didn't know or think of it this way!' or you know like seeing them, they connect vibrations and amplitude together, and those two things [are] both scientific... You can see that they are more and more convinced each time [about the connections between physics and music].

This reflection, coded as inbound Negotiated Experiences, about how enjoyable it is to see children at the end of the workshop connecting the concepts of music and physics, which is the Quavers to Quadratics domain. Therefore, the facilitator experience translates into insider accountability to the enterprise, which is fostering inward membership movement.

Community membership is the second most coded mechanism of identity overlapping with accountability to the enterprise (shown by the pink square in Fig. 4) and tracks how facilitators' confidence in the practices of the community impact their membership. This identity mechanism is related to elements of the structural design of the program; in the case of Science Theatre, this would be related to the community-built collection of demonstrations and show scripts along with the physical spaces that the group has to hold meetings. Science Theatre facilitators are undergraduate students from various different backgrounds and areas of study including both science and nonscience majors. Therefore, they have a considerable amount of content to master, as well as needing to learn how the demonstrations work and the phenomena behind them. For example, Liam, an undergraduate student whose background is not in physics, stated that he feels like a science person because he asks a lot of questions when asked if he identifies as a scientist:

But I was asking questions like, this dixie cup demo worked really really well. Does it have to be paired with the bed of nails? Could you do Bed of Nails with dixie cups and then have another part, just have the dixie cups demo. Because you could do that. That demo in and of itself is pretty great... I was asking a lot of practical questions where I'm like, in practicality, how can we make this work better? How can we explain this to where it makes more sense? 
In this quote, Liam is explaining that he is insistent in learning the content and practices of the program by asking his peers (community membership). Liam's questions show his growing knowledge of and confidence with the Science Theatre practices so this segment was coded as inbound accountability to the enterprise and inbound community membership. He goes on to explain that he cares more about making the experience better for the children and assuring that they do not leave with misconceptions even if they do not remember the concepts. He does this because he cares about the experiences the children have in the program and making science fun and understandable (part of the domain of Science Theatre).

Relationship between Local and Global (shown by the yellow triangle) and the insider subcode for accountability to the enterprise also frequently, as seen in Fig. 4. This mechanism relates to the connection between participation in the local community and participation in a broader (global) community of practice. Developing awareness of those connections requires a deep understanding of the domain of the local community and how it plays a role in a global perspective.

Across the programs the main theme identified in segments coded as accountability to the enterprise relates to the facilitators' wish to be part of a community focused on encouraging children to enter STEM fields. For many interviewees, being involved with Science Theatre provided a bit more than just a way to satisfy their physics curiosity, it provided a chance to share physics with others. For example, Grace, an undergraduate who has been involved in Science Theatre for several years, connects her interest and involvement in Science Theatre to her other identities and shares how this intersection is important to her:

I really like [Science Theatre] because it's like a chance to reach out and spread what you're passionate about, and I think that, especially as a woman of color, I want to see more people in science in general. Especially reaching out to young girls, young girls of color. Because, you know, science can be very monolithic and when you do outreach you get more types of people.

In this case, Grace's statement was coded as insider accountability to the enterprise because she is connecting her value of wanting to be a role model for women of color in physics within Science Theatre's mission to provide fun and engaging physics experiences for everyone. This portion of Grace's interview was also coded as inbound relationship between local and global because she is describing how her participation in Science Theatre can have a broader impact on the physics or science community at large, regarding issues of underrepresentation.

In the case of PISEC, the facilitators are already committed to the physics community and its value, given that they are mostly Ph.D. students working toward research that will add to understanding phenomena, so what PISEC allows them to do is communicate their passion for physics to children. As Evan, a physics graduate student, gives the following answer when asked why they first volunteered for PISEC:

I've kind of really grown to [where] I would do PISEC now even if my boss told me I didn't have to, just because I feel like it's a great idea to give back to the community and to kind of just be there as someone who can say 'hey, science is cool.' Because I didn't have anybody when I was that age telling me science was cool, so I feel like taking just an hour and a half out of your time, out of your day, and kind of just introducing the world of science to some kids is a good thing, is a fun thing to do.

This portion of the interview was coded as insider accountability to the enterprise and inbound relationship between local and global because there is an indication of a commitment to participate in the community, regardless of the implications and therefore perceiving the value of doing outreach. Evan is reflecting on how he would have liked the opportunity to participate in a program like PISEC as a child, and that is something that he can contribute now.

In Quavers to Quadratics, student interviews show that the facilitators hold similar views to their PISEC and Science Theatre counterparts around the importance of the program's values, as said by Seán, an undergraduate physics major:

I mean I have always been a big believer in outreach, and I think it's very important especially for scientists to do. Maybe I am biased because I am involved in science, but it's affected how I view it and I think I can put more emotion into it now because I [have] taken part in [outreach]... But I have always been a big believer in outreach so it didn't change that in any way shape or form but yeah I understand now there is more emotion behind that and how gratifying it can be.

This quote from Seán was coded as insider accountability to the enterprise and inbound relationship between local and global because he is explaining how he sees the value of the outreach community and how that intersects with the domain of the physics or science community. Sean is also narrating how participation in Quavers to Quadratics has reaffirmed the positive feelings related to being a member of the science outreach community.

\section{B. Intersection with mutuality of engagement}

Mutuality of Engagement is related to interactions between community members, the norms of those interactions, and how we recognize and are recognized as members of the community of practice. Therefore, it is expected it would have more interaction with the mechanisms of identity related to interactions with other members 


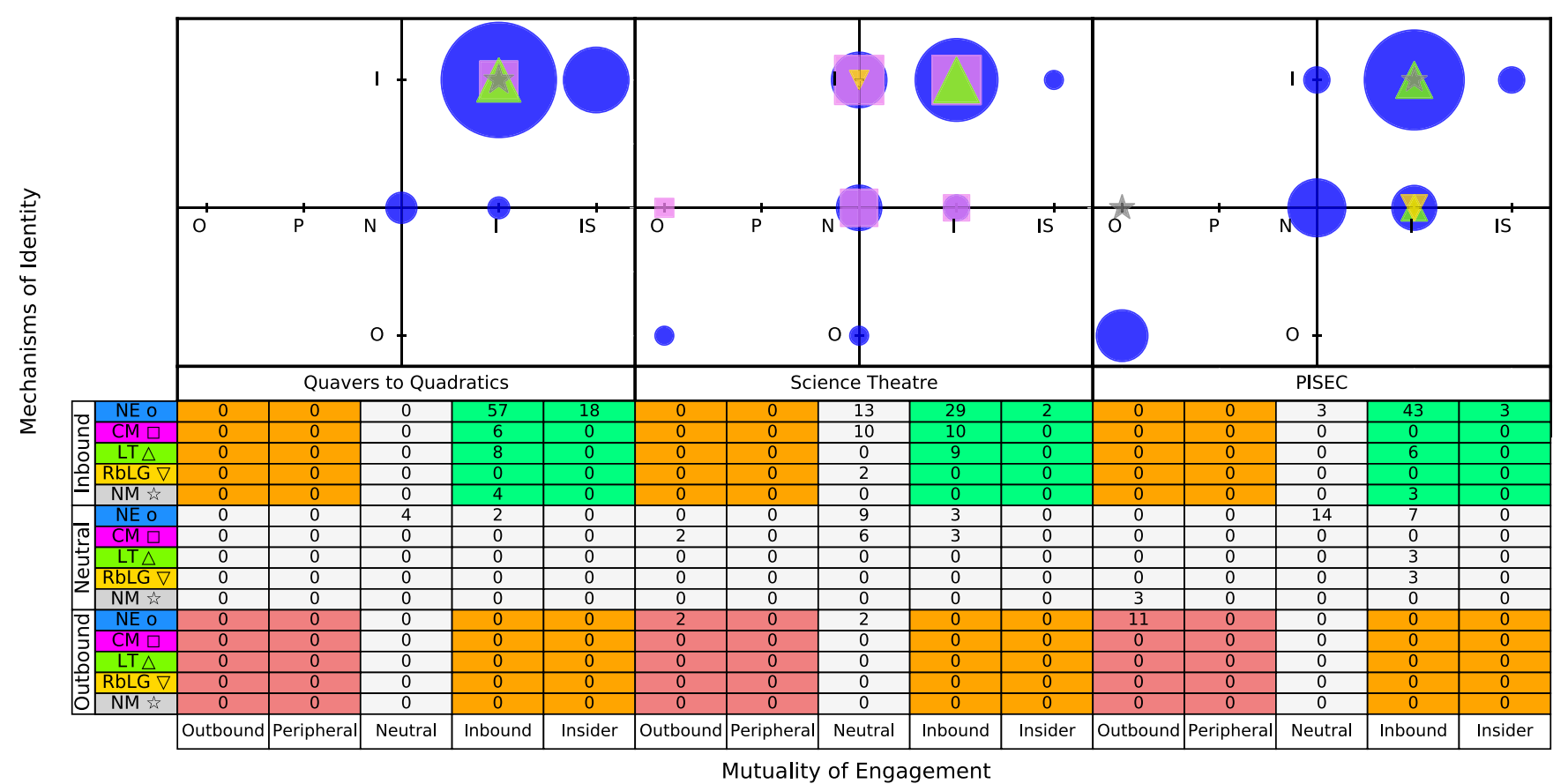

FIG. 5. The mechanisms of identity overlap with the mutuality of engagement. The frequency of the overlapping is represented by the size of the markers. The table mirrors the plot and shows the frequencies in each quadrant marked with different colors.

of the community such as negotiated experiences and community membership. This is confirmed in Fig. 5, where the majority of the interactions for all three programs are associated with these mechanisms of identity.

Negotiated Experiences (shown by the blue circle in Fig. 5) represents one of the most often mentioned mechanisms to build identity within the communities of practices observed here. The relationships with other members of the community, whether the children, partners, or peers, fosters and supports facilitators' membership. Feeling supported and recognized by their peers provided a stepping stone for newer members to venture into becoming more central members. The excitement and engagement children showed towards physics was a contagious feeling that reminded facilitators of their passion for physics and encouraged them to continue participating and become more central members of the community. This is consistent with other identity frameworks where being recognized as a member of the community has been proved to be one of the main aspects of building identity [13]. This is true for the programs' community of practice as well as the discipline's community of practice. Through participation in the informal physics communities, children and peers recognize the facilitators as members of the programs' community of practice and as experts of the discipline (physics) community of practice.

Moreover, the majority of the mutuality of engagementnegotiated experiences interactions are on the upper-right quadrant, meaning that negotiated experiences is fostering a movement inward for the mutuality of engagement dimension. There are some outward interactions of mutuality of engagement-negotiated experiences, mostly for PISEC and Science Theatre which are related to instances where facilitators were having a hard time engaging the children with the activities. However, the facilitators expressed that positive interactions would always outweigh the inevitable frustrations.

We observe that mutuality of engagement is the least coded community dimension for Quavers to Quadratics and Science Theatre. A reason for this smaller number of codes is related to identifying who is considered a member of the community of practice. In the case of Science Theatre and Quavers to Quadratics, the children are not regarded as members of the community, while for PISEC they are, which comes from the design of the programs. In PISEC, facilitators interact with the same group of children for $1 \mathrm{~h} \mathrm{a}$ week, for at least one semester, developing important bonds and relationships. The children are co-creators of the activities because they decide what they want to do and how, and the facilitator is there to provide support. The format of Science Theatre and Quavers to Quadratics means that children interact with the facilitators for a very short period of time and while there is some degree of participation from the children in how the activities developed during that time, there is more structure established by the facilitators on what will be done and how.

However, in both Science Theatre and Quavers to Quadratics, facilitators spend more time working together as they develop and practice the activities or during the trips. These interactions allowed facilitators to be supported 
by their peers when learning the norms, content, and practices of the program. In the case of science theatre, newer facilitators expressed a general sense of support by the officers and returners because all of the more experienced members were happy to provide guidance and help where needed. For example, when Daisy, an undergraduate student who is in her first year doing science theatre, is asked what has participating in science theatre meant to her, she talks about her interactions with other members:

I went with [Jean]-but she has been to other [Upper Peninsula] trips. She's super enthusiastic about all of the demos that we do, and it was really too cool to have her as my first person to have a show with, 'cause she taught me a lot.

This was coded as inbound mutuality of engagement and inbound learning trajectory because for Daisy it is clear that Jean's enthusiasm for the program and the mentorship that she provided helped Daisy feel supported and build her membership in the community.

For both Science Theatre and Quavers to Quadratics, the trips (to rural Michigan and rural Ireland, respectively) provided good opportunities for bonding outside of the regular practices of presenting the demos and facilitators mentioned how these interactions were positive in helping create connections inside and outside the community of practice. For example, Grace explains that bonds built between Science Theatre colleagues due to the time spent in the cars helped them create a rapport and trust that they relied on during the presentations causing codes of mutuality of engagement inbound and negotiated experiences inbound:

Because we'd spend a lot of time in the car [laughter]. It's like no way you can't bond when you're in the car that much and spending that much time with each other. And during shows, things happen, so if something goes wrong, you have to rely on each other.

Similarly, when asked about highlights of the Quavers to Quadratics Galway trip, Orla, an undergraduate physics major, expressed:

...in terms of like I guess the social side of things so it was really nice to bond as a group and I think we got really close with the Galway people. It just worked really well, yes it was good.

This was coded insider mutuality of engagement and inbound negotiated experiences as it shows that Orla developed relationships with her fellow facilitators and enjoyed the bonding process; viewing herself as a central member of the community had a positive effect on Orla's identity.

On the other hand, for PISEC, the most relevant interactions come from the personal relationships that facilitators were able to build with the children over a semester(s), with the majority (65\%) supporting an inward trajectory in membership. When asked about the most important thing gained from her involvement with PISEC, Lily discusses how interacting with the children and their enthusiasm makes her move to a more central position in the PISEC community but also influences her membership in the physics community by increasing her commitment to the domain:

And then also just like being with the students in PISEC and like being around their excitement and enthusiasm helps me be, like I said earlier, kind of like more excited about physics. Like wow, this is really cool, like this is why I love physics is because of these cool things that I get to learn with like middle school students.

These thoughts from Lily were coded as inbound mutuality of engagement and inbound negotiated experiences. She then continues to say what she has gained from her participation, "I guess like as far as PISEC goes, just feeling like I'm a member of this community, like the CU middle and elementary schools. This kind of like community connection, just feeling like I'm a part of that is a big thing that I gained I would say."

Furthermore, facilitators described how the interactions with the children came as a refreshing change of scenery and perspective, compared to their everyday physics community of practice. For example, Ava mentions how much she enjoyed having to work with a group of girls in PISEC because in her everyday physics $\mathrm{PhD}$ work, she has mostly male colleagues " $[\mathrm{I}] \mathrm{t}$ was great because my group was always girls, and I really loved that because like here I'm just interacting with males, males, males all the time." This was coded as mutuality of engagement inbound and negotiated experiences inbound as Ava's positive interactions with members of the PISEC community potentially moved her to a more central member of the PISEC community of practice and intersects with her physics community of practice.

Unlike in Science Theatre and Quavers to Quadratics, interactions with fellow facilitators were not discussed much in PISEC, possibly because facilitators only shared car journeys to and from schools and the occasional social event. However, other interactions that did affect PISEC facilitators' membership in both PISEC and the physics communities of practices were with their $\mathrm{PhD}$ advisors. For example, Evan, a physics graduate student, says "so it started out with my [research advisor] telling me that I should volunteer for PISEC, but as it went on, I really started to kind of enjoy the interaction with the students." This quote was coded for inbound mutuality of engagement and inbound negotiated experiences because it demonstrates that supervisors' support can impact the students' physics identity and their participation in the PISEC 
community. Unfortunately, some PISEC facilitators did not receive this support, like Ava, a physics graduate student, who said she "never really had a lot of positive [encouragement] — in fact my advisor was kind of like not that supportive of doing outreach, because he was like 'yeah it's good but you should get your PhD first' kind of thing" which was coded as outbound mutuality of engagement and neutral nexus of multimembership. However, Ava continued with PISEC as "it was really fun. And the kids were really enthusiastic, so that was great," which was coded with inbound mutuality of engagement and inbound negotiated experiences. Ava's experience shows how lack of advisor support made her doubt her participation in PISEC, but because she enjoyed working with the enthusiastic children she continued in the program.

\section{Interactions with negotiability of the repertoire}

Negotiability of the repertoire is associated with members' competencies and knowledge in the skills, practices, and norms of the community. Therefore, we expect that it would interact more with the mechanism of identitycommunity membership, which relates to how we learn the practices of the community through interactions with members, as seen in Fig. 6.

Community Membership is the most frequently coded mechanism of identity for Science Theatre and Quavers to Quadratics (39\% and 34\%, respectively). Both programs place emphasis on university facilitators becoming more central members of the community through learning how to do the physics activities. There is also an emphasis on the performative aspect, which is mentioned by Liam while in his first year with Science Theatre:

There [were] a lot of demonstrations where I kind of understood them. Like, when someone explained it to me, I'm like yeah, that makes sense. But it's a whole different thing, someone explaining this is how you derive $X$ as opposed to you explaining this is how you derive $X$.

This reflection was coded as inbound negotiability of the repertoire because Liam is explaining that there is a difference in understanding the content and explaining the content, especially to younger kids. He is moving towards becoming a central member as he develops those skills, and his identity within Science Theatre and science is growing as his competence with the practices develops, captured by the inbound community membership code.

The coded interactions that occur in the upper-right quadrants of Fig. 6 (inbound and insider codes) indicate that facilitators' identities are supported through participation in the practices of the community as well as learning the practices through interactions with other members. Ciara, an undergraduate student new to Quavers to Quadratics, shares the following when asked what she is gaining from being involved:

I'm getting a lot of confidence. Like personal confidence and the fact that [I] don't shy away from [it all, when] we're starting off. I didn't know [about] sound waves or frequency or anything like that. You have to just take the

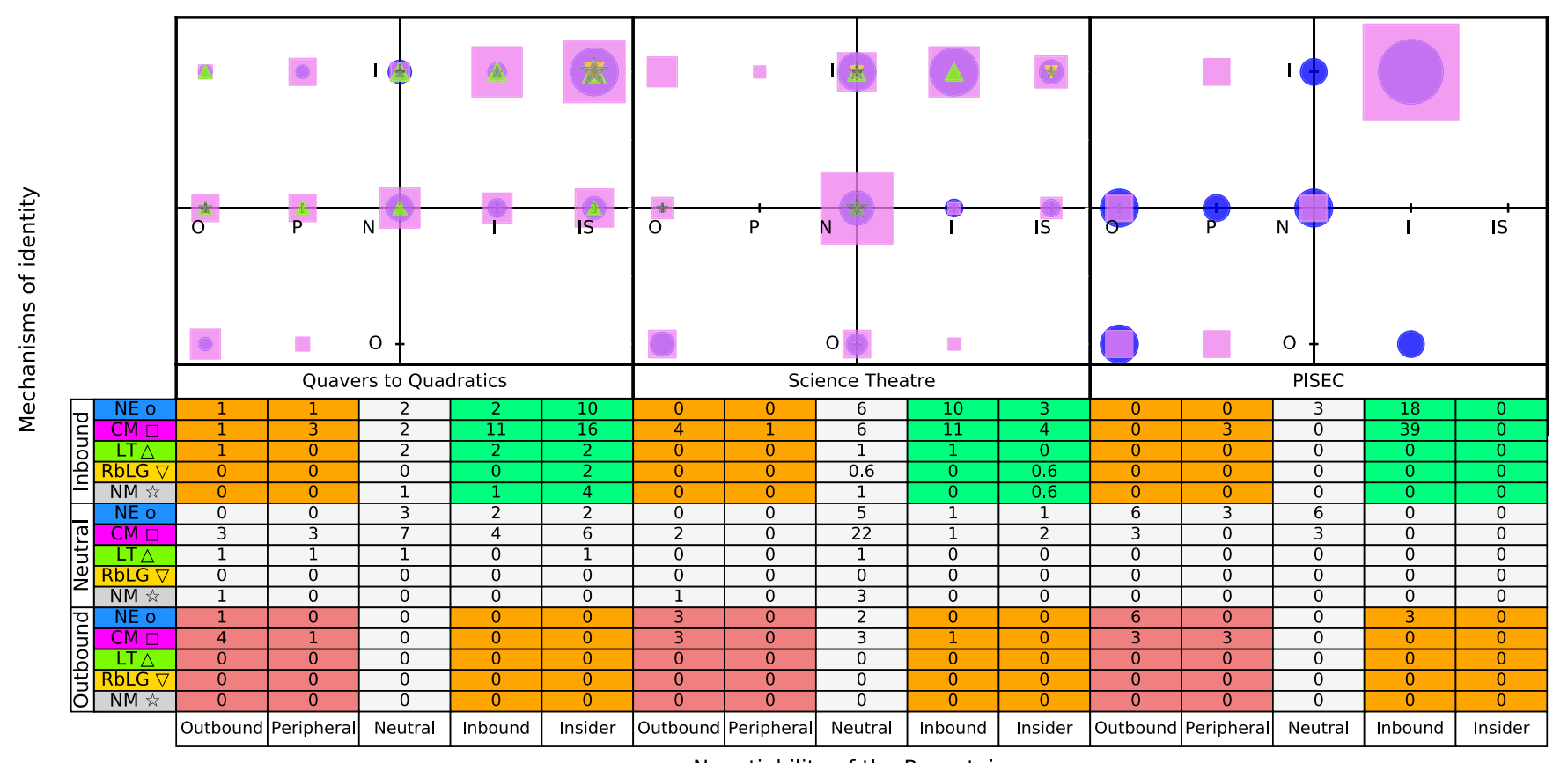

Negotiability of the Repertoire

FIG. 6. The mechanisms of identity overlap with the negotiability of the repertoire. The frequency of the overlapping is represented by the size of the markers. The table mirrors the plot and shows the frequencies in each quadrant marked with different colors. 
challenge and it's really rewarding to see the kids understand different concepts.

This was coded as inbound negotiability of the repertoire-Ciara's positive approach to learning the physics content allowed her to successfully teach it to the children, which in turn caused an increase in her personal confidence. This confidence boost produced an inward movement in her membership in both the Quavers to Quadratics community and the physics community (inbound community membership).

Science Theatre members also discuss their repertoire by speaking about how they have discovered better ways to communicate about the demos and activities unique to the program. For example, Jacob, an undergraduate student, explains that using physics jargon does not necessarily add to the understanding of the phenomena:

[W]e have another one, another Physics demo, that you just don't bring up mass, because mass is-you can say in all these equations, there is mass, but trying to get a little kid to understand what mass is, is not worth it. And all the mass cancels out anyway [laughter], so you're never going to be, 'Okay, we've done this experiment, having Sally spin around on the chair. Now we're going to do a massless spinning.' Like no, that's-so yeah, it's just figuring out what to bring up and stuff like that. And so I think as the week went on, I definitely got a better sense of like, okay, judging my audience and figuring out what they could handle.

This was coded as inbound negotiability of the repertoire because he is describing how through the process of participating and engaging in the practices of the community, he was developing more confidence and competence in those practices. Jacob's growing confidence affects his identity positively and so was coded as inbound community membership.

Negotiability of the repertoire represents only $22 \%$ of the community dimensions in PISEC (see Fig. 2 for details). Unlike in Science Theatre and Quavers to Quadratics, the PISEC facilitators are graduate physics students with a strong foundation in undergraduate level physics, of which the PISEC activities are based on. Also, the model of the PISEC program requires less performance than the other programs. Rather, PISEC activities are more exploratory and one-on-one, with facilitators working alongside children on hands-on activities to try to answer questions. Thus, in PISEC, facilitators do not need to act as experts in front of a crowd but rather as coaches with the children, which deemphasizes the importance of expert physics content knowledge. This feature is reflected in the absence of codes related to learning the content; facilitators instead focus on the practices related to learning to communicate the content appropriately for their audience, as discussed by Lily:
Well one thing, I think it's really important for physicists to be able to explain their work, and physics concepts in general, in a simple way to young kids or to people who are not familiar with physics, and that's really hard to do. So like when you first do PISEC and you have to [make a video for kids communicating about your research]-I think it just takes a bunch of practice I think. And I often find family members or friends who never learned physics will ask me stuff about physics, like 'what is physics, what do physicists do, what do you do?' and sometimes it's really hard for me to explain it in a way that is accessible. So, I think being involved in these types of programs is really helpful for that.

Lily's comments show a growing understanding of PISEC practices and developing confidence about how she can explain physics, coded as inbound negotiability of the repertoire and inbound community membership. She sees knowing how to communicate with the audience as an important practice for being a member of the PISEC community, but also identifies those practices as part of the physics community repertoire. Her improvement in those practices produces inward movement into both those communities.

Negotiability of the repertoire has the largest percentage of outbound codes for all three programs-representing about $20 \%$ of the overall community membership codes (see table in Fig. 2 for details). The outbound codes mainly reflect the challenge of developing practices that lie outside a facilitator's area of expertise. For example, in Quavers to Quadratics, Ciara, a facilitator with no science background, expressed "So we were actually a team of musicians put together but at first it was a bit daunting, with the science aspect", which was coded as negotiability of the repertoire outbound and community membership inbound. However, she then continues to say "actually it worked to our advantage because we found our own way and we kind of made our lesson plan to see the way that we were looking at things" which was coded negotiability of the repertoire insider and community membership inbound showing the development of Ciara's confidence with the Quavers to Quadratics practices. In Science Theatre, the large majority of outbound codes came from one student who did not have a science background; it is understandable that a particular student might find the practices more challenging than someone with prior knowledge of the content. In general, outbound codes tended to come from newer members of the community, demonstrating the need to support newcomers and scaffold their inward trajectory within the communities of practices.

\section{DISCUSSION}

Our analyses of the three presented informal physics programs indicate that there are particular structures and practices within these programs that can support physics 
identity development. It is important to us (and is a call for action within the field of informal science education [42]), that research results should be useful to practitioners working within informal spaces. Thus, in this section, we combine our practitioners' knowledge (which we used to establish the community of practice attributes of each program as described earlier) along with the findings from the coding to pull out themes that can suggest practical changes within existing or future informal programs.

Figure 7 shows several main themes that emerged across the three programs in our study. These structures are not comprehensive or necessarily applicable to all programs; however, we do think that they provide a starting place for connecting the communities of practice analysis to concrete aspects of program design and implementation. We discuss how the data connect to these structures, and how these structures may be used by practitioners to consider changes to their local programs.

A major theme across the three programs highlighted by the accountability to the enterprise code and the relationship between local and global code is the importance of having a program mission that aligns with the values of the facilitators. Across our data, facilitators expressed a strong desire to participate in informal physics programs as a way to have a physics experience outside of their coursework and research that aligns with their personal values. Many facilitators discussed their desire to participate in informal programs prior to joining them, some even selecting the university they attended based on the informal programs available. Facilitators were highly committed to sharing their physics knowledge and their passion for physics with younger students, especially girls and youth of color, or students who may not have access to physics due to rural settings or socioeconomic status. They talked about wanting to be role models through their participation. Furthermore, many facilitators spoke about how they did or did not have access to informal programs when they were young and how this inspired them to make sure others did have this opportunity. Thus, having a strong mission and communicating clearly about that mission is a key structural component of the programs in our dataset.

Another theme is that interaction with the audience is critical for positive identity development for the facilitators. We saw this theme within the intersection of the negotiated experiences identity mechanism with all three community dimension codes. Facilitators join a program because they

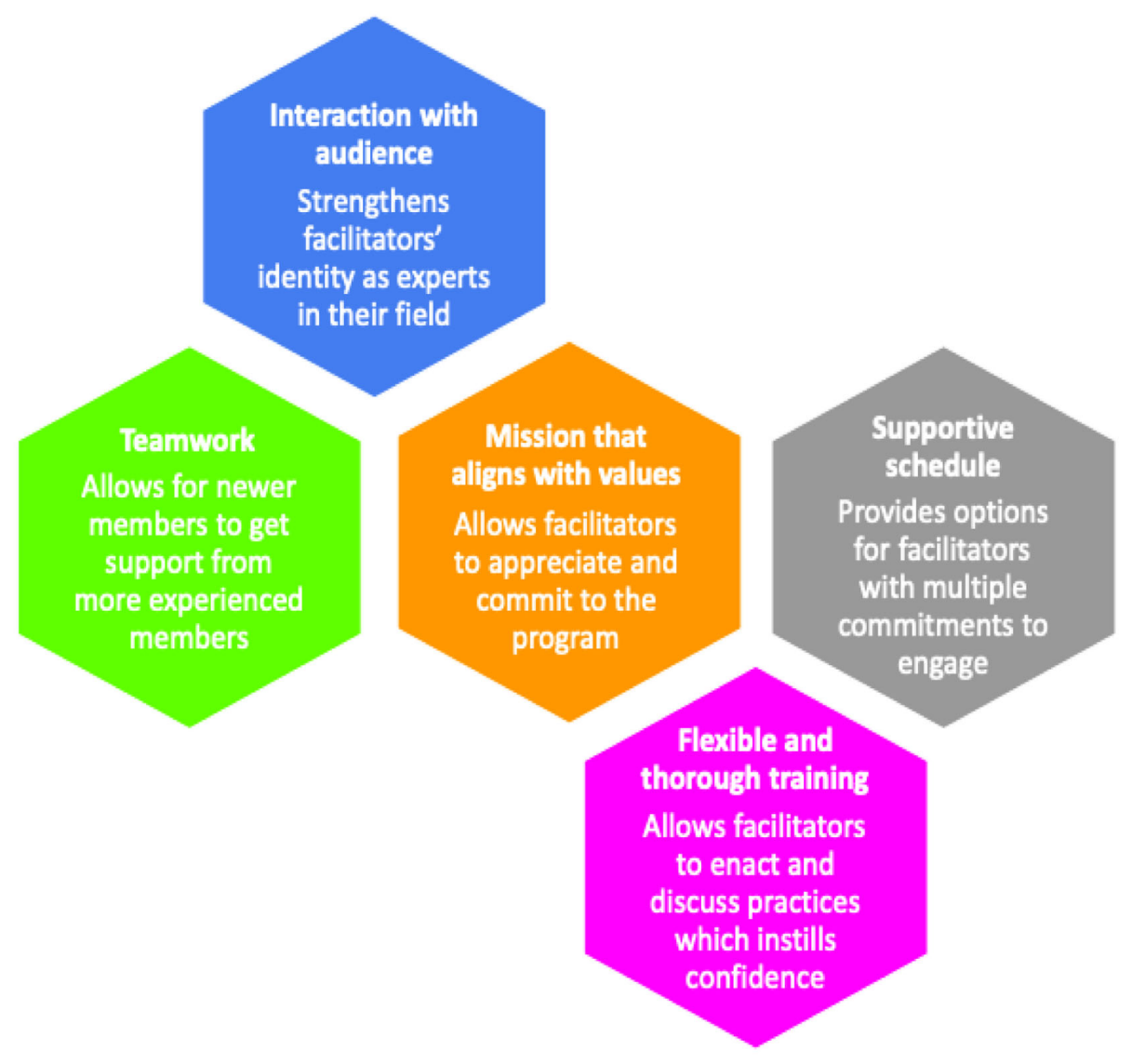

FIG. 7. Identified structures that support facilitators' identity based upon the interview analysis. These structures are general, and practitioners are encouraged to consider other program specific features. The color of each hexagon indicates the community dimension or mechanism of identity that each structure is connected to (e.g., teamwork is in green as it connects to mutuality of engagement). 
connect with the mission of the program and the mission aligns with their identities. However, it is their enactment of these missions through engagement with the audiences that moves them inwards in the community. Additionally, one result seen from our data set, which includes student facilitators being interviewed at multiple time points, is that more extended and deeper interactions with the audience may have been a bigger contributing factor to the development of facilitators' identities. In PISEC, facilitators really enjoyed building relationships with children over time doing co-constructed physics activities. In Quavers to Quadrics and Science Theatre, facilitators also feel a kind of relationship with their audience, especially in seeing the effect of their program on children. Additionally, when student facilitators feel like they are using their expertise to help others, they are more likely to move inbound in the community, similar to what has been shown in the formal setting with learning assistants [32,33]. Thus, our data show that the interactions between the children and facilitators are the most important aspect of the program for facilitators. This finding is consistent with other research in which recognition (both internally and through external validation) as a member of a community of practice can be a determinative factor for identity development $[7,11,12]$.

Another important program structure is a supportive schedule, since facilitators worry about fitting informal physics into their busy schedules. We saw this theme emerge from the nexus of multimembership and accountability to the enterprise codes, as some PISEC facilitators experienced conflict between their desire to participate in the program, their academic schedules, and the pressure they felt to devote most of their time to research. Our analysis showed the facilitators really wanted to commit to informal programs as they saw the benefits, but time away from work was a concern. This worry caused a negative impact on the facilitators' identities and led some to reconsider their involvement. Support for the informal physics program from leaders at their home institution (e.g., director of physics center, research advisors, professors in classes) was crucial for some student facilitators for inward movement in the physics community of practice because they perceived that public engagement is a practice that physicists should engage in. Practitioners of informal programs may be able to help with this issue by being aware of their facilitators' other commitments and trying as much as possible to work around them.

Flexible and thorough training is another structure we identify that stems from facilitators' negotiability of the repertoire and community membership. A frequently mentioned issue, especially for new facilitators, was the anxiety they initially felt learning the practices in the programs. For Science Theatre and Quavers to Quadratics this stress was mainly due to the performative nature of the programs' practices and the need to learn new physics content. The anxiety they felt about their perceived lack of knowledge sometimes negatively affected their identity development and their position within the community, both in physics and in the corresponding program. However, some facilitators struggling in this arena reported that training sessions and working with more experienced members of the community were helpful to overcoming this anxiety and actually developing their skills. Thus, flexible and thorough training that involves more central community members is a key structure to aid facilitators in increasing their confidence with content and practices.

Teamwork is another structure we found in Quavers to Quadratics and Science Theatre that helps ease anxiety and support the facilitators. We see this through the intersection of the mutuality of engagement code and negotiated experiences. Working with more experienced facilitators not only increased new members' confidence in themselves, it also moved the new facilitators inwards in the community because they felt supported. Additionally, the bonding between facilitators that was evident during both Science Theatre's (as mentioned by Grace) and Quavers to Quadratics' (as mentioned by Orla) trips caused positive identity development and further shows how the interaction between members of these groups is important.

\section{IMPLICATIONS AND CONCLUSIONS}

In summary, we have used the intersection of community dimensions and mechanisms of identity from the communities of practice framework to analyze three different informal physics programs. This application of the operationalized framework highlighted the opportunities for positive physics identity development among the student facilitators. Participating in informal programs supported their interest in physics, allowed them to be recognized as members of the physics community, and expanded and supported their participation in the practices of the physics community. From the coding, we identified some program structures that are key to moving members to more central positions in the informal physics communities of practice. These results are complementary to findings from previous studies that showed informal physics programs can provide growth opportunities for university physics students in specific physics practices-such as improved scientific communication skills [10] and pedagogical modalities [8,9].

The differences and similarities between these three programs were highlighted by the coding results, as discussed in the analysis, showing that the framework can adapt to the complexities of varied programs. As the framework simply requires an informal physics program to operate as a community of practice- a group of people working together (a community) to achieve a shared mission (a domain) through various activities (practices) - it can be used by practitioners to analyze their programs. We hope that this framework can serve as a valuable tool for both researchers and practitioners to further explore different types of 
informal physics programs and the ways in which programs can support student facilitators.

We also envision this operationalized framework being useful for many contexts outside of informal physics programs to analyze different aspects of physics identity development. There are many communities of practice in action in the daily life of a physicist. For example, the physics department of a university has a domain that includes furthering physics research and educating the physicists of the future, a community of physicists, and a practice involving giving lectures and running labs for students, conducting experiments and other research, participating in seminars and conferences, etc. This framework could be used to analyze how members of that community of practice feel supported (or not) with the continuous development of their identity as a physicist. Further, the framework can tweeze out structures and norms that can be crucial factors towards people moving more centrally in the community. Thus, physics departments may be able to use this information to make adjustments to foster more positive identity development for all of its members.

Informal physics programs are part of the physics community's practices. Involving physics students in public engagement with local communities is clearly something that is valuable not just to audiences but to the physics students themselves. Through research and evaluation of these programs, including the use of a community of practice lens, we will be better able to provide support for all participants who engage with physics outside of the classroom.

\section{ACKNOWLEDGMENTS}

We would first like to acknowledge and thank our study participants, who have shared their time, energy, and stories to contribute to this work, as well as all three programs (PISEC, Quavers to Quadratics, and Science Theatre) for being open to participating in this work. Additionally, we want to acknowledge Manuel Vazquez for his support on the initial stages of this study and Noah Finkelstein for his guidance and contribution to all of this work. This paper is based upon work supported by the European Research Council through Marie Skłodowska-Curie Actions Individual Fellowships (MSCA-IF) Project No. 794434, and NSF Advancing Informal STEM Learning Grant No. 1423496. Any opinions, findings, and conclusions or recommendations expressed in this material are those of the author(s) and do not necessarily reflect the views of the European Research Council or the National Science Foundation.
[1] National Research Council, Learning Science in Informal Environments: People, Places, and Pursuits (National Academies Press, Washington, DC, 2009).

[2] https://www.aps.org/programs/outreach/index.cfm. Retrieved November 1, 2020.

[3] C. Fracchiolla, B. Prefontaine, M. Vasquez, and K. Hinko, Is participation in public engagement an integral part of shaping physics students' identity?, in Research and Innovation in Physics Education: Two Sides of the Same Coin (Springer, Cham, 2020), pp. 225-238.

[4] C. Mullen, C. Fracchiolla, B. Prefontaine, and K. Hinko, Why it should be 'and' not 'or': Physics and music, in Proceedings of the 2019 Physics Education Research Conference, Provo, UT (AIP, New York, 2019).

[5] T. Williams, S. Hyater-Adams, K. Hinko, C. Fracchiolla, K. Nordstrom, and N. Finkelstein, The intersection of identity and performing arts of black physicists, in Proceedings of the 2018 Physics Education Research Conference, Washington, DC (AIP, New York, 2018).

[6] B. Prefontaine, C. Fracchiolla, M. Vasquez, and K. Hinko, Intense outreach: Experiences shifting university students' identities, in Proceedings of the 2018 Physics Education Research Conference, Washington, DC (AIP, New York, 2018).

[7] C. Fracchiolla, S. Hyater-Adams, N. Finkelstein, and K. Hinko, University physics students' motivations and experiences in informal physics programs, in Proceedings of the 2016 Physics Education Research Conference, Sacramento, CA (AIP, New York, 2016).

[8] K. Hinko, P. Madigan, and N. Finkelstein, Characterizing pedagogical practices of university physics students in informal learning environments, Phys. Rev. Phys. Educ. Res. 12, 010111 (2016).

[9] M. B. Bennett, B. Fiedler, and N. D. Finkelstein, Refining a model for understanding and characterizing instructor pedagogy in informal physics learning environments, Phys. Rev. Phys. Educ. Res. 16, 020137 (2020).

[10] K. Hinko and N. Finklestein, Informal science participation positively affects the communication and pedagogical skills of university physics students, in Proceedings of the 2013 APS April Meeting (AIP, New York, 2013).

[11] K. Hinko and N. Finkelstein, Impacting university physics students through participation in informal science, AIP Conf. Proc. 1513, 178 (2013).

[12] C. Fracchiolla, B. Prefontaine, and K. Hinko, Community of practice approach for understanding identity development within informal physics programs, Phys. Rev. Phys. Educ. Res. 16, 020115 (2020).

[13] Z. Hazari, G. Sonnert, P. Sadler, and M.-C. Shanahan, Connecting high school physics experiences, outcome expectations, physics identity, and physics career choice: A gender study, J. Res. Sci. Teach. 47, 978 (2010). 
[14] O. T. K. Pierrakos Beam, J. Constantz, A. Johri, and R. Anderson, On the development of a professional identity: Engineering persisters vs engineering switchers, in Proceedings of the 2009 39th IEEE Frontiers in Education Conference, San Antonio, TX (IEEE, Bellingham, WA, 2009) pp. 1-6.

[15] S. Hyater-Adams, C. Fracchiolla, N. Finkelstein, and K. Hinko, Critical look at physics identity: An operationalized framework for examining race and physics identity, Phys. Rev. Phys. Educ. Res. 14, 010132 (2018).

[16] S. Hyater-Adams, C. Fracchiolla, T. Williams, N. Finkelstein, and K. Hinko, Deconstructing Black physics identity: Linking individual and social constructs using the critical physics identity framework, Phys. Rev. Phys. Educ. Res. 15, 020115 (2019).

[17] H. T. Holmegaard, L. M. Madsen, and L. Ulriksen, To choose or not to choose science: Constructions of desirable identities among young people considering a STEM higher education programme, Int. J. Sci. Educ. 36, 186 (2014).

[18] P. W. Chinn, Asian and Pacific Islander women scientists and engineers: A narrative exploration of model minority, gender, and racial stereotypes, J. Res. Sci. Teach. 39, 302 (2002).

[19] K. Rainey, M. Dancy, R. Mickelson, E. Stearns, and S. Moller, Race and gender differences in how sense of belonging influences decisions to major in STEM, Int. J. STEM Educ. 5, 1 (2018).

[20] T. Williams, The intersection of identity and performing arts of black physicists, undergraduate thesis, Mount Holyoke College, 2018.

[21] C. Funk and K. Parker, Blacks in STEM jobs are Especially Concerned about Diversity and Discrimination in the Workplace (Pew Research Center, Washington, DC, 2018).

[22] L. R. Hausmann, J. W. Schofield, and R. L. Woods, Sense of belonging as a predictor of intentions to persist among African American and White first-year college students, Res. High. Educ. 48, 803 (2007).

[23] K. Rainey, M. Dancy, R. Mickelson, E. Stearns, and S. Moller, Race and gender differences in how sense of belonging influences decisions to major in STEM, Int. J. STEM Educ. 5, 1 (2018).

[24] Z. Hazari, P. M. Sadler, and G. Sonnert, The science identity of college students: Exploring the intersection of gender, race, and ethnicity, J. Coll. Sci. Teach. 42, 82 (2013).

[25] K. L. Lewis, J. G. Stout, S. J. Pollock, N. D. Finkelstein, and T. A. Ito, Fitting in or opting out: A review of key social-psychological factors influencing a sense of belonging for women in physics, Phys. Rev. Phys. Educ. Res. 12, 020110 (2016).

[26] E. O. McGee, Devalued Black and Latino racial identities: A by-product of STEM college culture?, Am. Educ. Res. J. 53, 1626 (2016).

[27] B. F. Albanna, J. C. Corbo, D. R. Dounas-Frazer, A. Little, and A. M. Zaniewski, Building classroom and organiza- tional structure around positive cultural values, AIP Conf. Proc. 1513, 7 (2013).

[28] D. Auguste, T. D. Ennis, S. L. Miller, and J. L. Polman, A Mixed-methods Study of Non-text Social Media Content as a Window into African-American Youth STEM Identities, in Proceedings of the American Society for Engineering Education Annual Conference \& Exposition, Salt Lake City, UT (2018), Paper ID \#21942.

[29] M. Hemmasi and C. M. Csanda, The effectiveness of communities of practice: An empirical study, JMI 21, 262 (2009), https://www.jstor.org/stable/40604647.

[30] E. Wenger, R. A. McDermott, and W. Snyder, Cultivating Communities of Practice: A Guide to Managing Knowledge (Harvard Business Press, Cambridge, MA, 2002).

[31] Etienee Wenger-Trayner and Beverly Wenger-Trayner, Introduction to communities of practice: A brief overview of the concept and its uses, 2015. Retrieved June 7, 2021, https://wenger-trayner.com/introduction-to-communitiesof-practice/.

[32] E. W. Close, H. G. Close, and D. Donnelly, Understanding the learning assistant experience with physics identity, AIP Conf. Proc. 1513, 106 (2013).

[33] E. W. Close, J. Conn, and H. G. Close, Becoming physics people: Development of integrated physics identity through the Learning Assistant experience, Phys. Rev. Phys. Educ. Res. 12, 010109 (2016).

[34] E. Wenger, Communities of Practice: Learning, Meaning, and Identity (Cambridge University Press, Cambridge, England, 1999).

[35] J. W. Creswell and C. N. Poth, Qualitative Inquiry and Research Design: Choosing Among Five Approaches (Sage publications, 2016).

[36] P. W. Irving and E. C. Sayre, Becoming a physicist: The roles of research, mindsets, and milestones in upperdivision student perceptions, Phys. Rev. Phys. Educ. Res. 11, 020120 (2015).

[37] J. Lave and E. Wenger, Situated Learning: Legitimate Peripheral Participation (Cambridge University Press, Cambridge, England 1991).

[38] L. Meerabeau, The nature of practitioner knowledge, in Practitioner Research in Health Care, edited by J. Reed and S. Procter (Springer, Boston, MA 1995).

[39] I. Shaw, Practitioner Research: Evidence or Critique?, Brit. J. Social Work 35, 1231 (2005).

[40] See Supplemental Material at http://link.aps.org/ supplemental/10.1103/PhysRevPhysEducRes.17.020134 for detailed description of methodology and analysis.

[41] S. Crowe, K. Cresswell, A. Robertson, G. Huby, A. Avery, and A. Sheikh, The case study approach, BMC Med. Res. Methodology 11, 100 (2011).

[42] Advancing Informal STEM Learning (AISL), National Science Foundation. Accessed October 6, 2021, https:// beta.nsf.gov/funding/opportunities/advancing-informalstem-learning-aisl. 